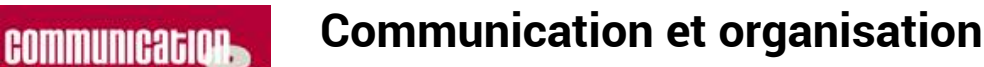

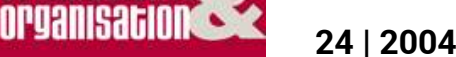

Coexister dans les mondes organisationnels

\title{
Situation et tendance du marché médiatique chinois
}

Yicheng Lao

\section{(2) OpenEdition}

1 Journals

Édition électronique

URL : http://journals.openedition.org/communicationorganisation/2932

DOI : 10.4000/communicationorganisation.2932

ISSN : $1775-3546$

Éditeur

Presses universitaires de Bordeaux

Édition imprimée

Date de publication : 1 mai 2004

ISSN : 1168-5549

Référence électronique

Yicheng Lao, "Situation et tendance du marché médiatique chinois », Communication et organisation

[En ligne], 24 | 2004, mis en ligne le 27 mars 2012, consulté le 02 mai 2019. URL : http://

journals.openedition.org/communicationorganisation/2932 ; DOI : 10.4000/

communicationorganisation.2932

Ce document a été généré automatiquement le 2 mai 2019.

(c) Presses universitaires de Bordeaux 


\title{
Situation et tendance du marché médiatique chinois
}

\author{
Yicheng Lao
}

1 Avec l'entrée officielle au sein de l'Organisation Mondiale du Commerce le 11 décembre 2001 la Chine a désormais adhéré à la mondialisation économique. Au cours des vingt années d'ouverture qui ont caractérisé « la Réforme » elle a toujours cherché à s'inscrire dans les critères internationaux du travail et elle a obtenu des résultats favorables. L'Organisation des Nations Unies pour le développement commercial indique dans le Rapport des investissements mondiaux en 2001 que les investissements directs à l'étranger globaux sont appelés à décroître sauf pour ce qui concerne la Chine. Le volume total du commerce extérieur de la Chine est de 509.8 milliards de dollars en 2001 soit trois fois plus qu'en 1990. La Chine occupe ainsi le $6^{\mathrm{e}}$ rang du commerce mondial alors qu'elle n'était que $16^{\mathrm{e}}$ en 1990 . De 1990 à 2001 les investissements étrangers se sont élevés à 510.8 milliards de dollars, dont 378 d'investissements directs.

2 Des changements historiques se produisent dans le domaine de l'économie en Chine. On aimerait ici étudier plus particulièrement la situation du marché médiatique chinois dans le cadre de cette mondialisation économique

\section{Entrée des groupes internationaux en Chine}

3 Au début des années quatre-vingt International Data Group (IDG) a pris pied sur le marché chinois des périodiques de la presse technique spécialisée, et a sorti douze magazines en collaboration avec la Chine, le plus connu étant Le monde Informatique. D'après Patrick J. McGovern président du conseil d'administration d'IDG, un milliard de dollars ont été investis en Chine durant les sept années suivantes. Le magazine américain Fortune a obtenu la permission de publication en Chine en 1999. Bertelsman AG société allemande bien connue, a créé à Shanghai un club de lecteurs pour les joint ventures : 1.5 million de membres en Chine et un chiffre d'affaire de 20 millions de yuans en 1999. En outre, des magazines américains célèbres comme Business Week et times sont également 
entrés en Chine. Dans le domaine des médias électroniques. Viacom Inc. a introduit le MTV comme un élément majeur du paysage audiovisuel chinois en collaboration avec la Télévision centrale de Chine (CCTV) ainsi qu'une centaine de télévisions locales: le programme est regardé dans 54 millions de foyers chinois. Depuis mai 2001. Nicklodeon canal éducatif, diffuse en Chine des émissions éducatives pour les enfants de huit à quatorze ans. Actuellement, elles sont diffusées sur cent canaux chinois répartis sur une vingtaine de provinces. En janvier 2001. AOI, Time Warner a acheté $85 \%$ des actions de CETV (China Entertainment Télévision) pour un prix de 100 millions de dollars. Cette chaîne d'information et de divertissement en mandarin sera disponible à partir de janvier 2002 dans la province de Canton, le 19 décembre 2001 le Groupe Star, filiale de News Corp. a signé un accord avec la CCTV la Société générale chinoise de télévision internationale et la Société du réseau de télédistribution du (Guangdong. En vertu de cet accord. Star a commencé, au début de 2002. à diffuser par câblodistribution 24 heures sur 24 dans la région du Guangdong une émission de variétés sur une nouvelle fréquence. En octobre 2001 la station de télévision Phénix, dont News Corp détient $37.6 \%$ des actions, avait reçu le feu vert pour diffuser dans la région du delta du Zhujiang les programmes de son canal chinois, son canal de cinéma était également disponible sur les réseaux câblés de Canton. Star TV et d'autres médias ont implanté leurs bases à Hong Kong et avec les stations de câble de 30 provinces et villes du continent, ont créé des émissions musicales, sportives, géographiques et de société. SUN-TV de Hong Kong, qui émettait pour tous les hôtels de trois étoiles et les quartiers relatifs aux étrangers, a été autorisé en octobre 2000 à diffuser à destination de l'ensemble continent chinois.

\section{Essor du réseau Internet}

$4 \quad$ Au début du XXI ${ }^{\mathrm{e}}$ siècle, grâce au progrès économique et scientifique, et aussi à l'élévation du niveau d'instruction du peuple chinois. Internet a pris un grand essor en Chine. Selon les statistiques de China Internet Information Center. il y avait 10.02 millions d'ordinateurs connectés au réseau et 26.5 millions d'usagers en juillet 2001. En un an ces deux chiffres sont passés respectivement à 16.13 millions et à 45.8 millions. D'après un reportage diffusé le 19 décembre 2002 par le Réseau du Peuple, en septembre 2002 deux mois plus lard, le nombre d'internautes avait atteint 54.34 millions, s'élevant au $3^{\mathrm{e}}$ rang mondial derrière les États-Unis et le Japon. Actuellement, presque tous les médias importants du continent chinois ont établi leur site web. Des statistiques montrent qu'il y a près de 300000 sites web sur le continent chinois. Internet est vraiment devenu le quatrième média en Chine.

\section{Renouvellement de l'idée de la communication}

\section{L'apparition de l'intérêt pour l'audience}

Depuis la Réforme et l'Ouverture, la Chine et les autres pays ont des contactes plus larges, les médias chinois commencent à réfléchir sur leurs idées en matière de communication, ils posent des questions telles que « qu'est-ce que les audiences préfèrent? » et «qu'estce que les audiences veulent savoir?». Par conséquent, le critère de la structure et du contenu se détourne fondamentalement vers l'orientation de l'intérêt pour l'audience. Cette transformation a débuté dans les années 1980 marquée par le groupe de lecteurs fondé par An Guang alors éditeur général du Quotidien économique, et elle a prospéré 
autour de 1991 concomitamment avec la vague de la sortie des journaux de week-end. C'est en 1995 au moment où les journaux métropolitains poussent comme des champignons que l'intérêt spécifique pour l'audience touche à son apogée.

\section{Le renforcement du particularisme de l'information}

Depuis les années 1980 avec le développement de l'économie et l'évolution de la société, les citoyens chinois manifestaient un désir croissant d'être informés et de disposer de la liberté de parole et de communication. Les médias abandonnent alors le mode idéologique traditionnel, et conçoivent une nouvelle forme de propagandes. La mise au jour de L'Orient (Donfang Shikong) sur CCI V en 1993 marque que la fonction " de défendre et d'envisager notre société " a succédé à celle "de civiliser et de propager ». Plus tard. Point focal (Jiaodian Fangtan) et Enquête de nouvelles (Xinwen Diaocha) ont été créés sur CCIV qui a donné une nouvelle image des journalistes chinois, en même temps qu'il redéfinissait et reconstruisait systématiquement le rôle des mass média. Simultanément, l'apparition des journaux métropolitains locaux ainsi que la naissance d'Internet ont renforçait le particularisme de l'information prôné par les médias d'une manière plus approfondie.

\section{Transformation de l'exploitation des médias}

\section{L 'exploitation à grande échelle.}

7 En janvier 1996 le Groupe de presse de Quotidien de Guangzhou a été crée, c'est le premier groupe de presse du continent chinois. En décembre 2000. le premier groupe provincial de la radio, du film et de la télévision a vu le jour - le Groupe de la radio, du film et de la télévision de Hunan. Actuellement, il y a plus de 40 groupes de médias autorisés par l'Administration de la presse et de l'édition et l'Administration d'Etat de la radio, du film et de la télévision. Ce qui marque que les médias chinois ont résolument œuvré à la transformation du fonctionnement et ont privilégié la compétence et la concurrence contre les abus de l'économie planifiée.

\section{L'opération du capital}

8 Actuellement, les médias chinois opèrent les capitaux de quatre manières :

9 - Exploitation en commun. En transférant les avoirs d'une certaine période, tels que droit d'exploitation et de distribution, chaîne, fréquence, rubrique et reproduction du contenu, une minorité des médias gagnent des investissements, ou bien créent et gèrent en commun des filiales avec des sociétés cotées.

10 - La filiale s'introduit directement en bourse. Les médias dégagent du capital, le réorganisent et créent une nouvelle société filiale par actions qui est la personne morale, laquelle s'inscrit ensuite à la bourse et collecte des fonds.

11 - La filiale contrôle la majorité du capital d'une société cotée. La filiale achète des actions d'une société cotée, puis elle entre en bourse afin contrôler et de réorganiser les actions : un capital solide est ainsi construit.

12 - Les sites Web des médias attirent des fonds selon le mode commercial. 


\section{Prédominance des activités principales et adoption de la stratégie de marque.}

\section{La prise en compte des besoins des audiences et de la qualité de la diffusion.}

13 Une mesure importante de la stratégie de marque consiste à privilégier les sources d'information et hausser la qualité de la diffusion en fonction des besoins des audiences. Les médias chinois prêtent désormais plus d'attention à l'acuité des problèmes et difficultés surgissant au fur et à mesure de l'évolution de la société, et essaient davantage à conquérir le marché par la Habilité des informations et la qualité de l'expression. Il s'agit de montrer une originalité des points de vue une vision particulière.

14 Ayant pour objectif de satisfaire les besoins d'audiovisuel et de lecture des audiences, les médias réalisent l'unification de la communication en faisant flèche de tout bois : recours à Internet, échange d'informations, critiques, achat du droit d'utilisation, etc.

\section{Le travail sur l'image et la valeur particulière du média.}

15 Une autre mesure de la stratégie des médias chinois est d'introduire la stratégie CI pour travailler sur leurs images. Depuis ces années. CCTV a créé des canaux d'information, d'économie, de cinéma et de sport, etc. dans le but de produire sa propre marque. En même temps, elle a consacré de nombreuses ressources humaines et matérielles aux affaires publiques et aux Travaux d'espoir (Xiwang Gongeheng) : ainsi se forgeait-elle son image de "station au service du peuple » avec pour effet l'accroissement des taux d'audience et l'augmentation des recettes publicitaire : en 2001 la publicité dégageait un bénéfice de 5.65 milliards de yuans.

16 Au cours de l'application de la stratégie de marque, un certain nombre demedias sont attentifs à l'apport particulier et irremplaçable de la communication. Ils recourent à des sources d'information originales et essaient d'attirer toutes les audiences par le contenu et la forme de leurs icontenus. Finance, un magazine dont les reportages revêtent un caractère unique et sensationnel on citera "les intrigues sournoises des fonds » ou « l'affaire de Yinguangxia » - fait école sur le marché très concurrentiel des médias de la finance, Point focal, une émission de CCTV bénéficie de la sympathie populaire, son autorité est incomparable, son rôle de superviseur et de critique est irremplaçable. On pourrait citer d'autres exemples.

\section{Segmentation minutieuse du marché médiatique et application à la concurrence}

\section{Le repérage des médias sur le marché et esprit marketing}

17 Au fur et à mesure que les médias s'orientent vers le marché et que se développe l'esprit marketing, les médias chinois sortent de la première étape fondée sur la probabilité de lire, de voir et d'écouter, et se tournent vers la nécessité de lire, de voir et d'écouter. La théorie de la segmentation minutieuse du marché, prônant que la segmentation, la cible et le repère sont trois en un. est devenue une base importante pour les médias qui veulent 
donner un service efficace. Se fondant sur la segmentation minutieuse du marché de la communication, les médias chinois parent mieux à toute éventualité, s'adaptent aux goûts des audiences, renforcent progressivement leurs compétences à saisir pleinement les conditions favorables, à éviter celles qui sont défavorables, et à formuler la meilleure stratégie marketing.

A présent, les médias chinois cherchent à éviter le gaspillage des ressources, à promouvoir l'unification et une concurrence sur le marché de la communication, et à diviser le grand marché en quelques branches ou filiales Visant à repérer les cibles. Ainsi, les points communs tolèrent les divergences sur le grand marché, et les divergences ont des points communs sur les marchés filialisés ce qui est le but recherché. Par exemple, en concurrence avec un vieux journal. Les Nouvelles de référence. Le Globe prend les jeunes pour cible, recherche les sujets de nature à répondre aux goûts de ses lecteurs qu'il parvient ainsi à fidéliser.

La plupart des médias sont très attentifs ù l'estimation de leurs spécificités afin de chercher le meilleur moyen d'étendre leur marché. Un vieux journal d'économie Les Références d économie, par exemple, après une comparaison entre la segmentation minutieuse du marché et son propre lectorat. a décidé de suivre le courant dominant dans le cadre de l'économie, au lieu de rapporter les affaires économiques quotidiennes. Il a ainsi trouvé su place sur le marché en fournissant des informations qui font autorité auprès de lecteurs qui travaillent dans les entreprises, les secteurs de l'économie du gouvernement, les écoles et les institutions économiques.

\section{La scientificité et la standardisation de la segmentation du marché médiatique}

La segmentation du marché médiatique s'engage progressivement dans une voie scientifique.

Enquêtes sociales et études de marché deviennent des outils pour les médias qui souhaitent connaître la position du marché et scientifiquement et efficacement, distinguer le marché médiatique segmenté. Nombreux soni les médias qui connaissent déjà le moyen d'étudier les tendances de consommation du marché global, les besoins des consommateurs potentiels, la division des produits médiatiques de ce domaine, la phase cyclique des produits médiatiques, le degré de popularité, lea demande annuelle, etc. Le changement du Journal Regional de Shen Zen en 2002 s'est fondé sur une connaissance mercatique obtenue par le sondage professionnel des lecteurs. Ce journal parvient ainsi à garder ses avantages traditionnels en incorporant un contenu tout nouveau et jette les bases pour son développement à long terme.

La scientificité de la segmentation marketing se manifeste aussi dans la maîtrise des différences substiles entre les différents segments marketing, ce qui permet la découverte de nouvelles divisions. L'Observateur de l'économie détermine précisément son marché fait de lecteurs de haut niveau et établit ainsi la position spéciale de son audience, selon sa connaissance de la situation chinoise des classes sociales et l'information qu'il a prise du marketing. Une année après sa fondation, ce journal devient un des trois plus grands hebdomadaires économiques de la Chine, avec le Journal chinois de Gestion et Rapport Economique du XXI siècle. 


\section{Ajustement de la structure industrielle, amélioration du niveau commercial} œuvre publique ou une organisation nationale. Mais dans une économie de marché, le média a non seulement ses propriétés politiques, culturelles, mais aussi industrielles et commerciales. Cette industrie comprend non seulement les blocs médiatiques au sens original, mais aussi une chaîne industrielle complète qui concerne des métiers comme l'agent publicitaire, le consultant informatique, les différents métiers de l'imprimerie et de la gestion de la production, etc. Avec le développement des techniques informatiques, l'apparition et l'expansion d'Internet façonne une plateforme informatique plus rapide et plus vaste pour le média. Elle est en même temps une plateforme commerciale et facilite l'intégration médiatique dans la vie sociale. Le média va donc certainement établir l'idée industrielle, construire la structure industrielle et le principe d'une compétition rationnelle, maîtriser un modèle commercial complet.

\section{L'ajustement structurel de l'industrie}

y aura des grands ensembles de presse et de grands ensembles audiovisuels. Dans le futur, il y aura peut-être des magnats des médias qui formeront une force prédominante dans l'espace marketing médiatique. Le marché médiatique connaitra sans doute "l'effet de Matthew ». C'est-à-dire le grand écart entre les riches et les pauvres. "Accumuler sa propre force le plus tôt possible pour s'agrandir et se fortifier dans la future compétition, c'est une tâche dure devant le média.»

Les modèles des différentes opinions et valeurs ont tendance à s'uni lier. Les journaux d'un parti ou d'une organisation tendront à s'adapter de plus en plus au marché, et les médias privilégieront les nouvelles dures et les paroles grandes dans une compétition forte.

\section{L'application intégrale aux différents secteurs}

Le premier secteur est celui de la publication et de la publicité. Maîtriser le marché par le secteur de publication et gagner de l'argent par le secteur de publicité, seront les principaux moyens de procurer des profits pour l'industrie médiatique.

deuxième secteur est celui de la gestion de la chaine de production médiatique. La stabilité et le fonctionnement efficace de la chaîne productrice médiatique est la clé qui garantit la qualité et l'efficacité de la production médiatique. La fabrication, la gestion et la diffusion des produits de l'imprimerie aussi bien que la fabrication, la gestion et la diffusion des produits de la radio et de la télévision, tout cela peut devenir un élément deprofit et de croissance.

Le troisième secteur concerne la production médiatique. La fabrication médiatique implique les supports, l'industrie de la fourniture des matériaux spéciaux et celle des matériaux communs. La presse ne fait pas exception avec la fabrication du papier et tous les métiers qui en découlent.

29 Le quatrième secteur est la gestion de la propriété. La réputation et la marque que le presse accumule dans son développement de long temps sont la propriété intangible, l'utilisation propre de réputation cl de marque ou bien l'utiliser comme capital dans un projet ou dans un domaine, devient un moyen d'élargir la gestion.

Communication et organisation, 24 | 2012 
30 Le dernier est la transformation de la gestion médiatique au média gestionnaire: les recherches, les consultations, les expositions, etc... ces services prolongés et de valeur ajouté seront les produits principaux de presse-média, l'industrie informatique et l'industrie d'idée seront le choix prochain du média. Le service informatique comprend essentiellement la recherche des diverses données, l'enquête informatique et le service consultatif. Aujourd'hui, le Presse Xinhua et le Quotidien du peuple ont constuit un databanque de recherche, cela permet à fournir le service aux usagers intérieurs et extérieurs par le satellite ou l'internet. Avec l'amélioration de l'intégration informatique médiatique, la perspective de ce domaine sera grande.

\section{Le renouvellement du système et l'accélération du rythme}

31 Renouveler le système d'investissement et de finance est une priorité. Le marché des capitaux est le centre de l'économie moderne, il possède les fonctions de financer, de former le système et de regrouper les capitaux. Le manque de capital, le retardement de système et la répartition irrationnelle des ressources sont les trois problèmes auxquels sont confrontés les médias chinois. À l'aide du marché des capitaux, par la gestion des capitaux, les médias de notre pays peuvent atteindre plus tôt leurs buts en termes de marché et de gestion et. en même temps, briser le système ancien de fonctionnement, perfectionner et affiner continuellement la structure de gestion en se référant au modèle international. D'un côté, pour prendre son envergure, le média doit conjoindre les affaires internes et externes de son secteur et faire une intégration des marchés : d'un autre côté, il faut absorber plus de capitaux attachés aux médias et les faire entrer en fonction sous forme de capital producteur, dans le but d'obtenir plus de capitaux et déposséder un plus grand marché.

Renouveler le système de gestion est une autre priorité. Le système gestionnaire administratif doit se transformer en système gestionnaire des entreprises modernes. La gestion transmédiatique est une stratégie ou un moyen généralement utilisé par les médias d'aujourd'hui, à travers la complémentation mutuelle des avantages respectifs, elle peut exploiter pleinement et utiliser les ressources comme l'information et la main d'œuvre du média, et produire des produits médiatiques sériels, qui satisferont les divers besoins des consommateurs, au coût relativement faible, arriver à la production et la vente d'envergure des produits médiatiques, saisir enfin une position forte au marché médiatique, lin brisant le système de « morcellement des secteurs ». le journal, la radio, la tele ${ }^{1}$ et la publication essaient activement de regrouper le cadre d'organisation gestionnaire médiatique et de prendre des mesures favorables, de briser les remparts artificiels entre les divers média, de construire les blocs transnationaux, transrégionaux et transmédiatiques, de réaliser le développement de «trans-type » des médias chinois.

On espère ainsi établir les codes du marché, construire l'environnement marketing d'une compétition raisonnable et offrir à chaque entité qui participe à la compétition l'opportunité de rivaliser à armes égales. Avec l'entrée de notre pays dans W IO. qui est basée sur les règles de l'économie de marché, la standardisation de l'environnement marketing et la compétition ordonnée sont nécessaires pour les médias chinois : il faut renforcer la surveillance de la compétition médiatique, perfectionner les lois et les règlements du marché, favoriser la victoire des forts dans une compétition loyale et la répartition raisonnable des ressources médiatiques, construire un système marketing médiatique unifié, ouvert, compétitif et ordonné. Avec le développement des réformes de 
la presse et l'amélioration du niveau marketing d'économie médiatique, la loi de victoire des forts va jouer un rôle décisif dans ce domaine, et on peut en attendre le développement complet et le mûrissage du marché médiatique.

\section{Engagement actif dans la compétition médiatique mondiale}

\section{L'établissement de "régions médiatiques spécifiques »}

La Chine considère déjà l'établissement d'une région spéciale au delta du Zhujiang comme une des décisions stratégiques de participation à la compétition médiatique globale. A présent, il n'est pas réaliste d'ouvrir tous les domaines médiatiques chinois et de s'engager complètement dans le processus de globalisation. Pour garantir l'utilisation pleine des opportunités apportées par la globalisation médiatique et résister à des risques prévisibles, la Chine a décidé d'établir " une région spéciale de média ». D’une part, elle peut ainsi créer " une période d'amortissement » pour l'entrée des médias extérieurs au continent chinois et fournir un champ véritable de bataille pour les médias chinois qui se trouvent devant la compétition internationale. D'autre part, elle peut accélérer les réformes du système gestionnaire du marché médiatique chinois, et construire un environnement favorable pour le rapide et raisonnable développement du média chinois.

\section{La coopération avec des blocs médiatiques transnationaux}

Il s'agit de coopérer avec des groupes transnationaux sous des aspects multiples, d'introduire, d'apprendre leur manière de gérer leurs capitaux, de comprendre leurs techniques et leurs expériences gestionnaires avancées, et de faire entrer nos produits médiatiques dans le marché international grâce à leur expérience et à leurs réseaux. Activement exercer des coopérations de multiples sortes entre les médias chinois et les groupes transnationaux peut non seulement, en quelque sorte, amortir le problème du manque de capitaux de la Chine, mais encore digérer et absorber leurs expériences gestionnaires avancées. En outre, par le service parallèle, la communication réciproque et l'alliance stratégique avec des blocs médiatiques transnationaux, les médias chinois peuvent réaliser l'expansion de leur participation à la compétition mondiale à un coût relativement faible. L'établissement de la coopération stratégique entre CCfV. AOL finie Warner et un groupe de presse en est une bonne illustration.

\section{La conjonction " sans soudure » entre les marchés intérieur et extérieur}

Dans la communication et la coopération mutuelles, on peut progressivement connaître les codes et l'état du marché des échanges internationaux des produits médiatiques, arriver à accélérer la construction de l'environnement du marché médiatique par la compétition et l'introduction des moyens el des expériences avancés. Il faut accélérer la mise à jour de la législation sur la presse et l'établissement du code des marchés des médias à l'intérieur, construire un bon environnement marketing au profit du développent médiatique à long terme, essayer de réaliser la conjonction « sans soudure » entre les marchés intérieur et extérieur. 


\section{L'amélioration des affaires presse-média pensées dans le contexte mondial}

37 Avec l'essor de l'internet l'environnement écologique et l'audience des divers média changent, les masses populaires ont de plus en plus de moyens d'accès aux informations et ont moins de dépendance par rapport à la presse et aux-médias traditionnels: en même temps, leurs aptitudes à la distinction et leurs capacités de critique des informations reçues augmentent. Le média doit changer progressivement les moyens, les modèles et l'expression traditionnels qu'il utilise, et transformer son idée et sa stratégie en temps opportun, réaliser la maximisation des avantages sociaux et économiques par la diffusion amplifiée et l'exploitation approfondie des ressources informatiques, basées sur les recherches d'audience et du marché.

Quant au reportage des événements imprévus (accidents, catastrophes), le degré d'ouverture du média est en train de développer. Celte année, deux explosions ont eu lieu successivement à l'Université de Beijing et à l'Université de Qinghua. les médias ont été capables d'en faire le reportage rapidement et de manière précise. Il en est ainsi, plus généralement, pour les accidents concernant la sécurité et l'intérêt publics. Le 20 mars cette année, à l'éclatement de la guerre en Irak. CCTV-l. CCTV-4 et la station Phénix ont fait un reportage en direct immédiatement. Leurs journalistes ont été envoyés aux frontières de l'Irak et à Bagdad. Cela a permis aux Chinois d'observer la guerre de leurs veux propres, et par la conjonction de reportages en direct et d'émissions d'analyses, nous avons exprimé notre voix propre dans la compétition médiatique pendant cette bataille importante.

\section{L'union organique d'internationalisation et de régionalisation}

En même temps qu'introduire et apprendre les moyens et les systèmes avancés, il faut se conjoindre organiquement avec la situation chinoise, et faire attention à la digestion, à l'absorption et au renouvellement. 